\title{
Two-Way Traceability using Smart Tags (QR): DL Tags
}

\author{
Sivasankari. S, Dinah Punnoose, Jaya Kalyan. T, Jagadeesh. P, Monendher. N
}

\begin{abstract}
The main goal of this project is to provide proof of goods from the start and their path through the supply chain along with preventing duplication and low-cost exploitation of goods. The network between the company and its suppliers can be described as a supply chain to manufacture and distribute a specific product to the final customers. This network encompasses numerous operations, persons, organizations, knowledge and resources. So, in order to helps the costumers and manufactures by bringing the transparency and avoiding the duplication. The Quick Response code $(Q R)$ is generated at the final stage of the production by combing the data from the various blocks of data such as from the raw materials to the dealers. This is a web-based application where every entity gets registered. Every raw material, manufactured sub product is to be given unique id and the data regarding that has to be stored in block of data. Everyone is provided with login and an automated unique id are generated at every stage so that all can be combined to obtain a final Quick Response code (QR) which the customer scans by Android application to get the details about the product.
\end{abstract}

Keywords: Supply Chain, Quick Response Code, Ethereum Block Chain, Traceability, Distributed Ledger Technology, Tag Duplications.

\section{INTRODUCTION}

In a supply chain management system, the smart tags (QR) are used in order to store the product details [3]. By that the consumers can also track the products during entire life cycle [1]. Product consumers have no knowledge about the data's that is provided in the smart tags by the smart tag creators and stack holders are true or not. The Distributed Ledger tags (DL-Tags) solution provides us a decentralized, which will give privacy and helps us to verify the Smart Tags across the lifecycle of a product at a low cost [4],[1]. The solution uses the popular distributed ledger technology and the Ethereum block chain to initiate and mediate the interactions between

Revised Manuscript Received on May 15, 2020.

* Correspondence Author

Sivasankari.S*, Department of CSE , ,Hindustan Institute of Technology and Science, Chennai, India. E-mail: kmiruthu@gmail.com

Dinah punnoose, Department of CSE,Hindustan Institute of Technology and Science, Chennai, India. E-mail:dinahpunnoose@gmail.com

T.jaya kalyan, Department of CSE , ,Hindustan Institute of Technology and Science, Chennai, India E-mail: kalyan.thoompati@gmail.com

P.Jagadeesh, Department of CSE , ,Hindustan Institute of Technology and Science, Chennai, India. E-mail: pavulurijagadeesh148@gmail.com .

N.Monendher, Department of CSE , ,Hindustan Institute of Technology and Science, Chennai, India, India. E-mail: monendher.n@gmail.com .

(C) The Authors. Published by Blue Eyes Intelligence Engineering and Sciences Publication (BEIESP). This is an open access article under the CC BY-NC-ND license (http://creativecommons.org/licenses/by-nc-nd/4.0/) the stakeholders during the transfer of a product from one phase to another phase between the stakeholders. The paper outlines the solution for DL-Tags and also provides a cost analysis and the record of all Ethereum blockchain transactions implemented. The proposed scheme offers proof of the origin of the product and its path across the supply chain, while avoiding tag distortion and duplication and tag manipulation. This is among the first recorded practical solutions for supply chain management using Distributed Ledger tags (QR code) and Internet of Things (IoT) [1],[2],[3]. The IOT devices involved here are simple printer to print the unique QR code and also the camera to scan that QR code [2].

\section{DISTRIBUTED LEDGER TECHNOLOGY}

DISTRIBUTED LEDGER TECHNOLOGY (DLT): DLT is a system which is used to record group of transactions and the details of transactions between two untrusted parties and these transaction details are stored in many places as like a record entered in log books in many places. The most advanced characteristics of distributed ledgers are immutable and they are cryptographically secured, censorship resistance and resistant with Iot devices, distributed centralized management, and does not need for a third party which is centralized, i.e. which is not intermediate. In another way, an organization does not need to take care between the disputed and should take care in part of preserving a global reality that is respected by all the stakeholders and the parties who do not trust each other because they implicitly don't know each other. DLT is also suitable and can be used for the tracing and keeping record of the ownership of digital assets [8], and its most prominent and the best application is the network of bitcoin. Moreover, DLT used of cryptocurrency for transferring ownerships using Ethereum or bitcoins because an entry in the ledger could be extended to hold arbitrary data. A digital ledger should store the details of transaction in an open ledger which is like a record book for keeping a global state [6]. Transactions serve as inputs that points the state and the shift of the state; therefore, the ledger can generally point to be interpreted as a state machine based on transactions. There are many types of data systems for holding of the ledger. One of those specializations of DLT, which is currently well referred in literature and often used for the clear meaning of the DLT, which is the data structure of blockchain. Since the blockchain-based public ledger and hyper ledger implementations are currently the most mature and we also use the type Ethereum block chain to implement the DL-Tags solution [12]. Bitcoin and Ethereum are examples of stable implementations of reference blockchains. New blockchain technologies

Blue Eyes Intelligence Engineering

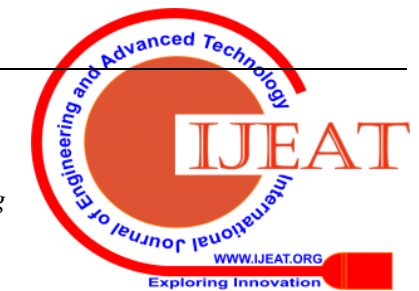


are implemented, but it was unstable and without sufficient development resources during the development process of DL-Tags.

\section{Ethereum Block Chain}

Ethereum is known as an public, which is open sourced and blockchain-based and distributed computing platform. Which uses the smart contracts and the proof of stakes for its processing. We are using the Ether to record and operate and initiate the transactions between the various entities in the supply chain using smart contracts which we had written. Ether is a type of cryptocurrency like bitcoins which are created by the Ethereum network as a reward for computations performed by mining nodes, and is the only and the most used currency accepted in paying transaction fees. Ethereum provides a virtual computer which is decentralized [12].

Like all the other blockchains, Ethereum also has Ether-ETH, a native cryptocurrency. ETH is mere digital money. Have you heard about Bitcoin [6],[5], ETH has a lot of the same characters. This can be submitted directly to everyone else in the world, and is solely digital. ETH's supply is not dominated by the government or by any corporation-it's decentralized, and scarce. People worldwide use ETH to make purchases, either as a store of value, or as collateral. Unlike other blockchains, however, Ethereum can do much more. Ethereum is according to our needs programmable, so developers can use it to create new kinds of applications [10]. Ethereum community is the most active Blockchain community and world's largest. This includes key protocol musicians, mining organizations, developers, academics, grandmothers, founders of the ETH, gamers, software creators, anarchists, 500 companies and as of now.

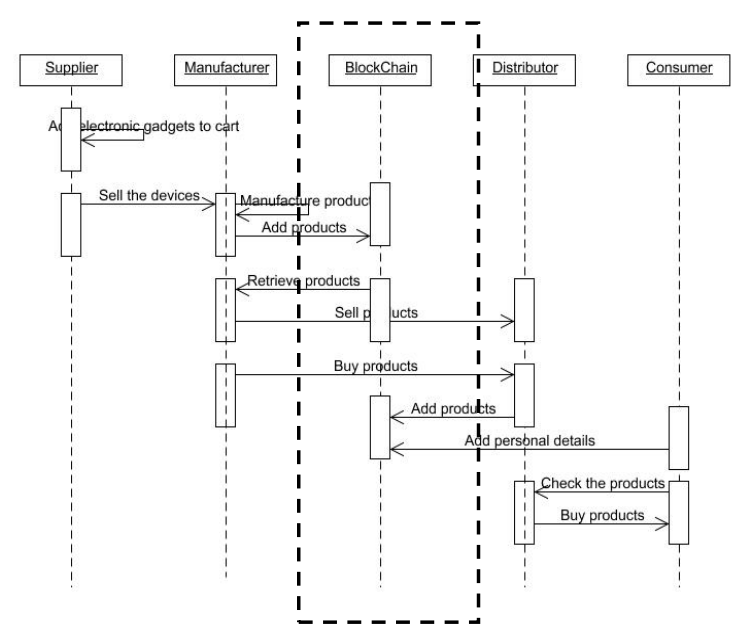

\section{Blockchain Technology}

By using decentralized database system and by using cryptographic hashing for security purposes we can call it as Distributed Ledger Technology, which makes the history of any digital assets unalterable and transparent. We are creating various blocks to store the data of transactions that are happening in the supply chain between various entities. A Google Doc and MS Excel are the classic examples of block chain technology where it can be logged and modified in many Systems [7].Whenever the document which we had created and shared can be accessed by everyone we can build and share a group of files instead of copying files in to the system.it can be used mainly for transferring the files. This establishes a decentralized distribution chain, which simultaneously allows everyone access to the text. No one is locked and should not wait for anybody to update and we can update also, while all modifications to the report are reported in real time, making changes fully clear.

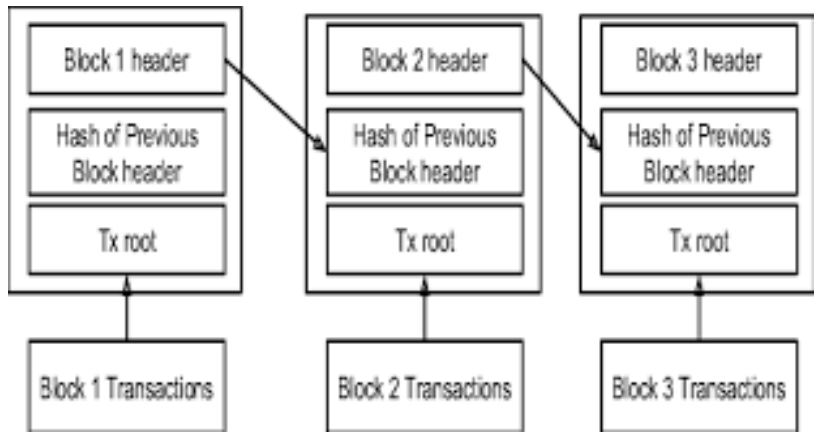

\section{QR CODE}

The name QR code (Quick Response code) is a type of matrix barcode (or two-dimensional barcode) which was first developed for the automotive industry in Japan in 1994.It is created for time saving and to reduce the human effort for storing a data for a product. A barcode is a machine-readable optical sticker, containing information on the object to which it is attached by using the machine we can read the data in the QR code and can be validated this is used for faster processing and reading [2]. In practice, QR codes often contain data that point to an application or a website for a locator, can be used for tracking, identifier. A QR code generally uses four standardized encoding modes (byte / binary, and kanji, numeric, alphanumeric) to efficiently and quickly store data [3]. Due to its easy readability and greater storage capacity while compared to standard UPC barcodes, the Quick Response system became popular and widely used outside the automotive industry for Brand monitoring, object recognition, time tracking, record management and general marketing applications and websites. A QR code typically consists of a white color background set which will have dimensions of a rectangular grid of black squares. Which can be read by a camera-like imaging system, and processed using Reed - Solomon error correction before the image can be properly interpreted. The necessary data are then eZxtracted from patterns present in both horizontal and vertical image components [2].

\section{Advantages of using $Q R$ code:}

Quick Response code is quite easy to generate cost effective and can be scanned using various devices like scanner, QR reader and even by the mobile camera.

\section{SUPPLY CHAIN}

A supply chain is basically a network between the company and its suppliers for manufacturing and delivering a particular commodity to the final purchaser. This network encompasses numerous operations, persons, organizations, knowledge and resources.

Published By:

Blue Eyes Intelligence Engineering

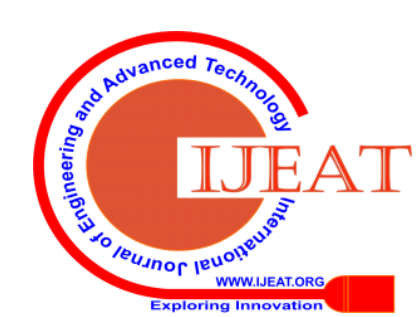


The Situation of the Covid-19 made us realize how important is the supply chain management and how weak is our current supply chain management. The figure below represents the supply chain for manufacture of a laptop.

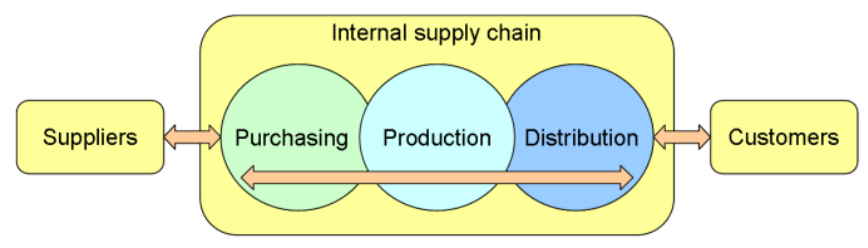

\section{TRACEABILITY}

Using the Distributed Ledger Technology every transaction and process can be easily monitored and it can be done even from customer side by tracing the raw materials and also in other way for manufactures to trace the customer who has received the product. In case the keyboards fitted for laptops at specific time are faulty they can be easily identified even by the customer as they know who are the manufactures of keyboard and when it's done so the recalling of units can be largely reduced. So, by bringing the more transparency it is made east to trace either from the customer side and also from the manufacture side. Even a specific complaint is received by the manufacturer from the customer such as mousepad from laptop manufacturer can get all the details of specific mousepad supplier so that he can procced with further action.

\section{TAG DUPLICATION}

Smart tag duplication can be done in many ways during a product life cycle if the seller is greedy to get more money and to marketize, lower-quality goods [3]. The seller duplicates the smart tag of an costly product and can paste it on a cheap product to get maximum profits. This fraudulent retailer can sell the fake products to other stakeholders or end users, without using the Distributed Ledger tags solution. Successive stakeholders or product users may eventually recognize the problem by detecting multiple queries from the TIS framework for this specific product item. It will, however, be hard to test which stakeholder committed the deception. The DL-Tags solution prevents this kind of fraud, as all stakeholders who use DL-Tags in their tag manufacturing System when we use the scanner to scan the tag then we will be able to find the duplicate product because we will use the change of ownership in this process. So that we can easily find the duplicate product if the tag is manipulated because the data which is retrieved will not match with the product data.

\section{METHODOLOGY}

Our proposed system uses the DL-Tags (distributed ledger technology) to provide a decentralized, privacy-conserving, and verifiable management of Smart Tags during the life cycle of a product. The solution relies on data collection IoT technologies and stores Blockchain product-related data, which is a private, decentralized data storage layer. DLT allows the global, append only, data structure to be maintained by a group of mutually untrusted participants in a distributed environment [5],[10]. i.e., if a consumer wants to buy a product which is duplicated by a fraudulent retailer by changing the data's in the smart tags. In case if a distributed ledger technology is used change is detected and products with duplicated smart tags will not be sold regularly. Here whenever a transaction is made between different participants, the legal agreement will be signed in the smart contracts. And the transaction details will be stored inside the Decentralized Blockchain Network. We are using private Blockchain for faster processing of transactions [5] if we are using public Blockchain then the time for updating the each and every transaction in all Nodes will takes much time. We are also using the QR (quick response) code rather than RFID because the QR codes can be easily generated cost effective and also the data from the QR code can be retrieved by scanning through and ordinary mobile phone camera that makes it less complex and more user friendly.

\section{TECHNOLOGIES USED}

$>$ J2EE (JSP, Servlet), JavaScript, HTML, CSS, AJAX.

$>$ Hibernate Framework

$>$ MVC Pattern (Model-View-Controller Pattern)

$>$ Design Pattern

$>\quad$ Spring (for starting block chain servers)

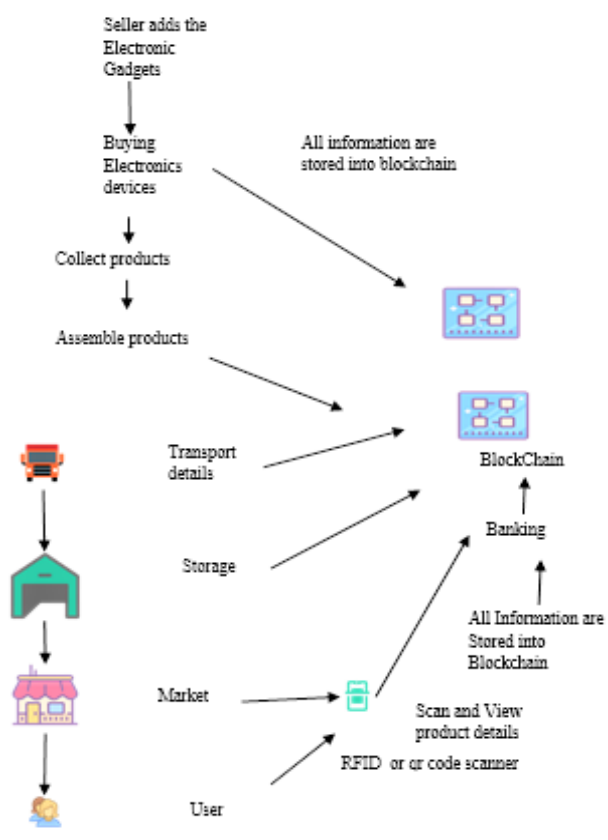

VIII. RESULT

By using this proposed system, we can obtain all the details related to the particular product (staring from the raw materials used for that). This is done from the costumer side by scanning a QR code generated by manufacturer using the application. Also the supply chain also be managed starting from the raw materials purchase to product purchase as we create separate login details and platform between the raw material manufacturer manufacturer and distributer they can all exchange the information regarding the goods and finalize the deals which are once confirmed gets stored along with time stamps.

Published By:

Blue Eyes Intelligence Engineering

\& Sciences Publication

(C) Copyright: All rights reserved.

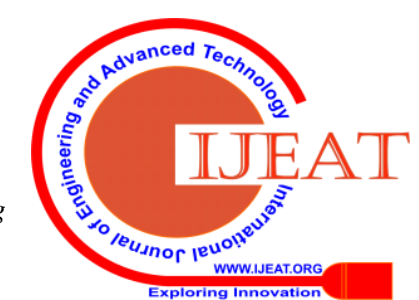


This is a stage in the Fig 9.1 as above-mentioned process where distributor is requesting the goods from the manufacture. This is manufactures module where he is monitoring requests received.

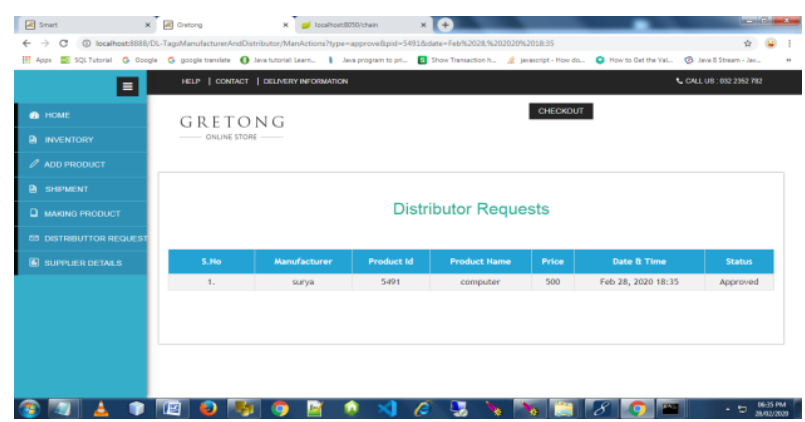

Fig 9.1

so that costumer gets every detail at the end while purchasing the product. The below here in the Fig 9.2 is output for costumer after scanning the QR through the application. So here the tracing gets easy and can be done in two way the manufactures can approve request and also track them while the costumer can also know every detail of the product along with time stamps.

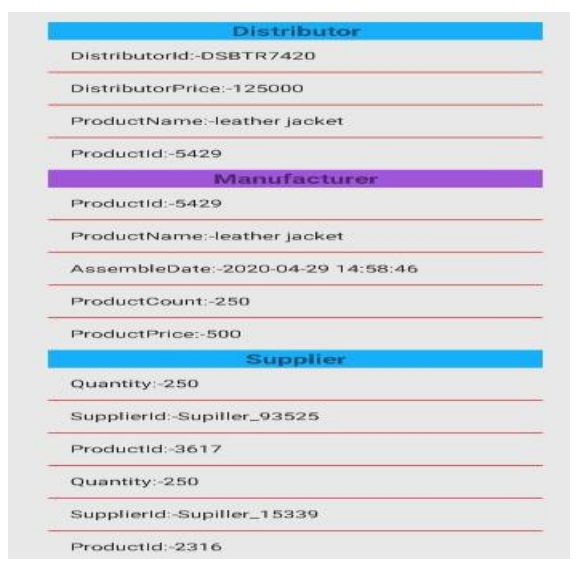

Fig 9.2

\section{CONCLUSION}

We conclude here that our proposed system uses DL-Tags (distributed ledger technology) to provide decentralized, privacy-conserving and verifiable management of Smart Tags during the lifecycle of a product. The solution relies on data collection IoT technologies and stores Blockchain product-related data, which is a private, decentralized data storage layer. DLT allows global, append only, data structure to be maintained by a set of mutually untrusted participants in a distributed environment. i.e., if a consumer wants to buy a product which is duplicated by a fraudulent retailer by changing the data's in the smart tags. In case if a distributed ledger technology is used change is detected and products with duplicated smart tags will not be sold regularly. Here whenever a transaction is made between different participants, the legal agreement will be signed in the smart contracts. And the transaction details will be stored inside the Decentralised Blockchain Network [12].

\section{REFERENCES}

1. M. Christopher, “'Logistics and supply chain management: Strategies for reducing cost and improving service (second edition)," Int. J. Logistics Res. Appl., vol. 2, no. 1, pp. 103_104, 1999.

2. M. S. Ali, M. Vecchio, M. Pincheira, K. Dolui, F. Antonelli, and M. H. Rehmani, "Applications of blockchains in the Internet of Things: A comprehensive survey," IEEE Commun. Surveys Tuts., to be published.

3. S. Georgoulas, S. Krco, and R. van Kranenburg, "TagItSmart_SmartTags for unlocking business potential," IEEE IoT Newslett., Nov. 2017. [Online]. Available: https://iot.ieee.org/newsletter/september-

2017/tagitsmart-smarttags-for-unlocking-business-potential.html

4. G. Zyskind, O. Nathan, and A. S. Pentland, “'Decentralizing privacy: Using blockchain to protect personal data," in Proc. IEEE Secur. Privacy Workshops (SPW), May 2015, pp. 180_184.

5. O. Svein, Beyond Bitcoin Enabling Smart Government Using Blockchain Technology (Lecture Notes in Computer Science), vol. 9820. Cham, Switzerland: Springer, 2016, pp. 253_264. [6] A. Narayanan and J. Clark, “Bitcoin's academic pedigree," Commun. Acm,

6. vol. 60, no. 12, pp. 36_45, 2017.

7. P. Franco, The Blockchain-Understanding Bitcoin. New York, NY, USA: Wiley, 2014, pp. 95_122.

8. F. M. Ben£iథ and I. P. .arko, “Distributed ledger technology: Blockchain compared to directed acyclic graph," in Proc. IEEE 38th Int. Conf. Distrib. Comput. Syst., Jul. 2018, pp. 1569_1570.

9. S. Nakamoto, Bitcoin: A Peer-to-Peer Electronic Cash System. 2008, p. 9. [Online]. Available: https://bitcoin.org/bitcoin.pdf

10. V. Buterin, “A next-generation smart contract and decentralized application platform," White Paper, 2014. [Online]. Available: https:// blockchainlab.com

11. B. Group and J. Garzik, Public Versus Private Blockchains. Part 1: Permissionless Blockchains. Washington, DC, USA: Bitfury Group, 2015, pp. 1_23.

12. G. Wood, “Ethereum: A secure decentralised generalised transaction ledger," Ethereum \& Ethcore, Ethereum Project Yellow Paper 151, 2014, pp. 1_32.

\section{AUTHORS PROFILE}

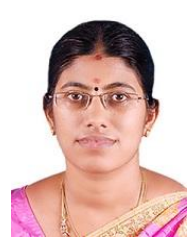

Dr. Sivasankari .S Received her $\mathrm{PhD}$ in Computer Science from Anna University, Chennai ,India. She is currently working as an Assistant Professor (SG) in Computer science department at Hindustan Institute of Technology and Science, Chennai, India. She has 8 years of teaching experience. She has published more than 10 research articles in leading journals, conference proceedings. Her research interests include Data Mining, Ontology, Bio-informatics, Artificial Intelligence and Machine Learning.

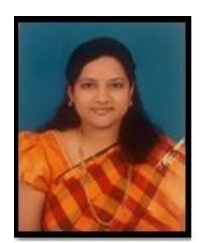

Dinah Punnoose received her Ph.D. in Computer Science and Engineering from Pusan National University, Busan, South Korea. She is currently working as an Associate Professor in computer science department at Hindustan Institute of Technology and Science,Chennai, India. She has published more than 30 research articles in leading journals, conference proceedings. Her research interests include computer network and machine learning.

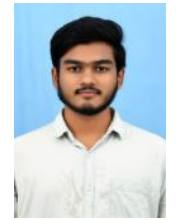

T. Jaya kalyan a final semester computer science student studying Bachelors of Technology in Hindustan Institute of Technology and Science,Chennai, Tamilnadu. I'm interested in C language and an active coder at GUVI. I am also looking ahead to work with Tech Mahindra. I have also done some certifications related to AI and Soft skills. 
P.Jagadeesh a final semester computer science student studying Bachelors of Technology in Hindustan Institute of Technology and Science,Chennai, Tamilnadu. I'm interested in Java language and an active coder at Hacker rank. I am also looking ahead to work with Tata Consultancy Services in the role of assistant software developer. I have also done some certifications in C, Java.

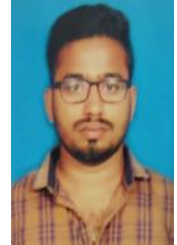

N.Monendher a final semester computer science student studying Bachelors of Technology in Hindustan Institute of Technology and Science,Chennai Tamilnadu. I'm interested in Java language and an active coder at GUVI. I am also looking ahead to complete the masters. I have also done some certifications in, Java.

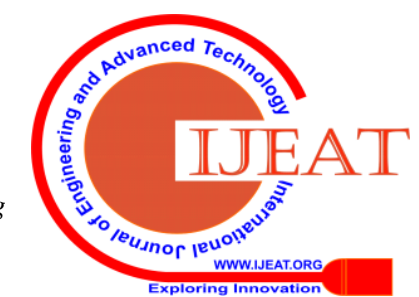

